\title{
Performance Assessment of Ultrasound Kidney Images using De-speckling algorithms
}

\author{
Harsha Herle \\ Research Scholar, Department of E\&IE, RV College of Engineering, \\ Affiliated to VTU, Bengaluru, Karnataka 560060, INDIA \\ harsha@rvce.edu.in \\ http://www.rvce.edu.in \\ Dr. K V Padmaja \\ Professor, Department of E\&IE, RV College of Engineering, Affiliated to VTU \\ Bengaluru, Karnataka 560060, INDIA
}

\begin{abstract}
The key concern that occurs in non invasive Kidney stone diagnosis using Ultrasound (US) imaging is speckle noise, as it reduces the diagnostic quality of images, required for further medication. In this work, different preprocessing filters like Median, Adaptive median, Weiner and Wavelet domain filtering are applied to both normal and kidney stone US images, with results showcase, Neigh Sure Shrink is preeminent for kidney stone US images . Objective feature assessment of the different preprocessing filters are evaluated for noise variance from 0.01 and 0.08 along with level of decomposition in Neigh Sure Shrink against the parameters like Signal to Noise Ratio (SNR), Peak Signal to Noise Ratio (PSNR), Root Mean Square error (RMSE), Mean Squared Error (MAE) with the investigational outcome shows the preeminence of projected filters over the existing methods. Further, the segmentation method allows bifurcation of the US image to detect the kidney stone on basis of Region of Interest, followed by determining the presence of Centroid and area for kidney stone US images. The Graphical User Interface allows easiness in locating the area of kidney stone for kidney US images.
\end{abstract}

Keywords: : Ultrasound (US) Image Adaptive filters; Wavelet domain filters; Signal to Noise Ratio (SNR); Peak Signal to Noise Ratio (PSNR); Root Mean Square error (RMSE); Mean Squared Error (MAE); Graphical User Interface(GUI).

\section{Introduction}

In recent years, the rapid growth of kidney abnormalities has gradually increased among patients of different age groups. Ultra Sound (US) technique is commonly used imaging system for the analysis of patients laid low with diseases associated with kidneys. US images are accepted due to the factors like low cost, portability, non-ionizing nature and monitoring of patients vital internal organs in real time. US image is recorded by penetrating high frequency (usually of order greater than $1 \mathrm{MHz}$ ) signal into the human body using a transducer. The reflected US waves from kidney tissues are received by the same transducer and projected onto a (2D or 3D) computer screen. During this process, US images is more prone to speckle noise- multiplicative noise. This noise is generated during US image acquisition process, when transmitted and reflected US wave intervene with each other. These speckle noise appear as dark and bright spots in the image, thus they are difficult to analyze and making diagnostic interpretation hard. To overcome this, speckle de-noising is applied for US imaging to improve the above mentioned drawbacks [1].

Mathematically, in US imaging, the speckle noise is modeled with multiplicative noise and given below:

$$
b(m, n)=a(m, n) * n(m, n)
$$

Here, original image $a(m, n)$ is multiplied with $n(m, n)$, the non-gaussian noise to obtain the speckle image $b(m$, $n)$. where, $m$ and $n$ represents the spatial position of the image.

Generally, de-noising methods are for additive noise, logarithmic conversion is applied to change multiplicative noise to additive noise for a speckle noise:

$$
\log b(m, n)=\log a(m, n)+\log n(m, n)
$$

The $\mathrm{n}(\mathrm{m}, \mathrm{n})$ is an additive noise component with zero mean. 


\subsection{Related Work:}

Medical imaging plays a significant role in detection and identification of diseases related to human subjects, medical image processing also have wide range of scope, thereby researchers and scientists have contributed significantly over decades [2].Removal of speckle noise from US images is a major field and many algorithms have been developed and differ in the basic methodologies. Of which, the most repetitive works involve, using detection of speckles and applying filtering or otherwise, filtering all the pixels, irrespective of losing the informative pixels. Two most common models, that are used to detect the speckle are, statistical or classification model. The main objective in statistical modeling is to remove the noisy images by concentrating only on statistical features from training data and later obtain the parameters of interest. Initially, statistical filters such as Weiner filter [3], adopted filtering in spectral domains, finds applicable only for additive noise [4]. In order to deal with the multiplicative noise, Jain model, can be applied [5], wherein, the logarithmic of the image is obtained, converts multiplicative noise to additive noise, and then applies the Weiner filter, but this process is tedious, as it takes more time for conversion and depends on the size of the image. A new adaptive filter was proposed and helps in significant improvement in sharpness of edges in US images with better smoothing [6]. A region based segmentation method is applied to kidney along with Gabor filter, resulting in removing of speckle noise, smoothen the image with histogram method to improve the quality of the image [7]. The experimental results demonstrate reduction upto $85 \%$ of speckle noise focusing on only few parameters. Generally, Adaptive filters use, Kaun filter [8], Lee filter [9] and Frost filter[10], and are advanced compared to temporal filters in removal of speckle noise. The other forms of adaptive filters used for speckle reduction are arithmetic mean filter to process homogeneous regions in US image and non linear median filter for edge pixels [11].

Much of the works is focused on estimating the optimal threshold value to separate the noise from US images, where a new method called Empherical Mode Decomposition (EMD) was used to decompose the image into a set of intrinsic mode functions (IMFs) with a surplus. The resultant image was smooth with reduction in speckle noise. This EMD method was for non stationary and non linear process [12]. Other thresholding methods like hard thresholding, Bayes SURE shrink soft thresholding, Visu-Shrink, Sure-Shrink, and Bayes thresholding for speckle reduction was also used [13]. The Visu shrink method is based on wavelet shrinkage and uses over smooth images. The Bayes shrink gives promising results in Mean squared Error (MSE) over visu shrink, all these methods are based on soft thresholding, with the input value is shrunk to zero by the amount of thresholding, whereas, in the hard thresholding, the input is retained to same value, if it is greater than threshold, else retained to zero[14]. Compared to other methods, Bayes threshold is based on maximum likelihood estimation. Fischer Discriminant Analysis (FDA) and bi dimensional EMD can be applied for 2D ultrasound images, to find the optimum value, which helps in noise reduction [15], extension of the work can be also focused on applying similar works to 3D images [16]. The experimental results of the proposed system exhibits improved result in reduction of speckle noise, edge preservation, visual quality and minimized Mean Square Error (MSE).

Other prominent method in removal of speckle noise is thresholding method with two level decomposition where the B mode scanned images were used in the experimentation to the accesses the parameters like SNR,PSNR, MSE and QI [16]. Another method to reduce the speckle noise base on multiscale imaging with increased accuracy can be seen improved accuracy [17]. Further, results demonstrated about $80 \%$ reduction in speckle noise, but loss of image features are caused due to the presence of bright and dark speckles in the image. [18] In proposed region based contrast enhancement, which mainly employs region based segmentation in which the initial seed point was auto generated, here segmentation is used in segregating background and foreground area, once it is segmented, foreground area will be enhanced there by getting a better contrast.

It[19], also focused on similar segmentation method of both kidneys, based on the experimental results obtained, the kidney dimension can be anticipated. Further experimentation resulted in presence of kidney abnormalities such renal calculus, multi cystic kidney in these images.

In classifying the abnormalities in kidney, statistical methods like Grey level Co-occurrence matrix (GLCM) or Run Length Matrix(RLM) were used for Support Vector machine reaching an accuracy of $85.8 \%$. [20]. Furthermore, the classification accuracy is improved upto $98.8 \%$ by using two level set segmentation methods with Artifical Neural Network (ANN) architecture [21]. Recent advancements in US imaging have revealed the way to increased interest in removal of the speckle noise from the medical images using different algorithms, without reducing much of the diagnostic information [22]

The current works aims to find the best preprocessing filter for detecting kidney stones in US images. The preprocessing filters that are discussed are compared in this works for US images related to kidney abnormalities, for parameters like SNR, PSNR, RMSE, MAE. Moreover, this work also estimates the best noise value to be well thought, when using different preprocessing filters like, median, Adaptive median, Weiner, and Wavelet transform by considering the above statistical parameters like SNR, PSNR, RMSE, MAE, for removal of speckle noise. 
The next step is to apply segmentation method like, threshold and Region of Interest method with morphological operation to detect the kidney stone abnormalities for further classification.

The final step allows positioning of the exact area of kidney stone located based on RoI and Centroid method. A Graphical User Interface (GUI) developed in this work will support the medical doctors to treat the patient to next level.

\section{Research Method}

\subsection{Proposed Design:}

Figure 1 depicts the system basic block diagram to detect kidney stone detection system from US images. At first, the US image (normal/kidney stone) is obtained from the radiologist, unwanted information like anatomical information, removed by binary threshold method. The noisy image filtered by using different filters as discussed in earlier section. The preprocessed image is segmented by threshold method and morphological operation [23] to detect RoI in kidney [24].

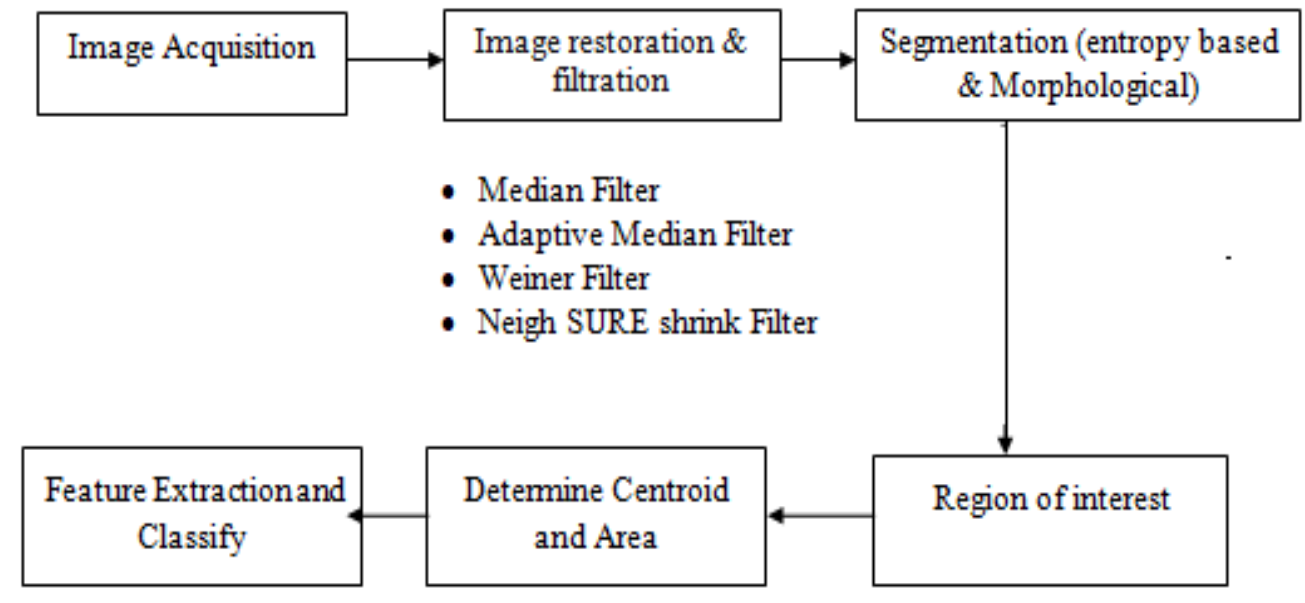

Figure 1: Block diagram of Kidney stone detection using US images

Furthermore, the statistical features are extracted from normal and abnormal images to classify the kidney abnormalities [25]. If the abnormalities are detected, then the centroid of the abnormal image is projected to find the area of kidney abnormal region. The detected abnormalities will provide information for medical doctors, to take the medication to next level. All the preprocessing methods, classification and detection of area are carried out in MATLAB simulation environment.

\subsection{Preprocessing filters.}

\subsubsection{Median filters:}

Median filtering are non-adaptive filter that adjust the filter coefficients based on data within a rigid moving window, and summons between the removal of speckle noise and the potential to preserve image details. They are linear statistical filter, works with the principle of replacing the current pixel value in US images into the median value in a neighborhood, w. This filter is advantageous for the reason; they don't reduce the intensity of the US image, as the brightness values are taken from original image. Secondly, edges of the images are redundant. The mathematical related in median filter is characterized by equation 3 given below.

\subsubsection{Adaptive median}

$$
y[a, b]=\operatorname{median}\{c[m, n],(m, n) \in w\}
$$

This filter works with the three step methodology of which the primary step is to detect the noise in US Images, secondly, adaptively estimate the window size based on the number of noise pixels in the window. Finally, determine the weight of each non-noise point in filtering window and remove noise points by means of weighted median filtering algorithm. This method is advantageous compared to other preprocessing filters, as it preserves the edges of its high frequency parts of an image.

\subsubsection{Weiner filter}

Another common preprocessing filter that finds application in US image is Weiner filter that works on the principle of blurring and removal of additive noise simultaneously by performing best possible substitution between inverse filter and noise smoothing [26] and is optimal in terms reduction in Mean Square Error. They are advantageous compared to other methods due to, efficient in removal of speckle noise, but, over smoothens the boundaries on vital image features, as compared to other methods. The frequency domain equation of Weiner filter is characterized by the equation 4 given below, 


$$
\mathrm{W}(\mathrm{f} 1, \mathrm{f} 2)=\frac{\mathrm{H} *(\mathrm{f} 1, \mathrm{f} 2) \mathrm{Sxx}(\mathrm{f} 1, \mathrm{f} 2)}{|\mathrm{H}(\mathrm{f} 1, \mathrm{f} 2)|^{2} \mathrm{Sxx}(\mathrm{f} 1, \mathrm{f} 2)+\mathrm{S} \beta \beta(\mathrm{f} 1, \mathrm{f} 2)}
$$

Where $\operatorname{Sxx}(\mathrm{f} 1, \mathrm{f} 2)$, and $S_{\beta \beta(\mathrm{f} 1, \mathrm{f} 2)}$ are the power spectra of original and denoising image. $\mathrm{H}(\mathrm{f} 1, \mathrm{f} 2)$, is the blurring filter.

\subsection{Wavelet filtering}

The majority of the techniques developed for speckle reduction are traditional filters in spatial domain. These filters are elementary and rapid as they have certain restrictions like susceptible to the shape and size of the filter window [27]. A larger window size leads to over smoothing of images and if the smaller window size, smoothing ability of the filter will decrease and the reduction in speckle noise is not accurate. A square window is mostly used, which will lead to rounding off corners. In order to overcome the limitations of spatial filtering, wavelet domain filters are proposed in this work.

\subsubsection{Wavelet Domain Filtering}

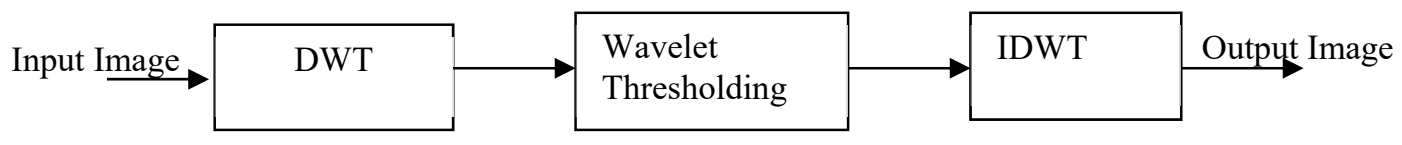

Figure 2: Block diagram of Wavelet domain filtering

Another preprocessing method used in speckle noise reduction is Wavelet Domain filtering as shown in Figure 2, where, input image is subjected to Discrete Wavelet Transform(DWT), followed by defining the wavelet coefficients, finally computing the output image by applying Inverse Discrete Wavelet Transform (IDWT) to obtain the de noised estimate. The prominent method used, exceptionally better results is to use Neigh Sure shrink method.

\subsubsection{Neigh SURE shrink}

Neigh SURE shrink is the adaptive filter based method works on the principle of thresholding technique, combining both soft thresholding and hard thresholding, and only deals with binary values[28]. In this method, a fixed threshold value is considered based on the window size, $\mathrm{W}_{\mathrm{s}}$ and variance value, is calculated using the equation 5 ,

$$
\sigma^{2}=\frac{\operatorname{median}\left(\left|\mathrm{w}_{\mathrm{s}}\right|\right)}{0.6745}\left(\mathrm{w}_{\mathrm{s}} \in\right. \text { subband LL) }
$$

The smooth or low frequency components are approximated from father wavelet and high frequency components are resembled from mother wavelet. A $3 \times 3$ sized window is moved all over the image to be filtered, where the surrounding pixel value is averaged over the central pixel. Mean and variance is applied over the decomposed image to supress the speckle noise from US image based on the thresholding value, when the pixel value crosses the threshold value then that pixel value becomes 1 or 255 and if the pixel value is below the threshold value then that pixel value becomes 0. The Neigh SURE Shrink uses a suboptimal universal threshold and identical window size in all wavelet subbands, whereas the improved version of it determines an optimal threshold and neighboring window size for every subband by the Stein's unbiased risk estimates as given in Equation 6 below.

$$
\left(\mathrm{T}^{\mathrm{s}}, \mathrm{k}^{\mathrm{s}}\right)=\arg \min { }_{\mathrm{T}, \mathrm{k}} \operatorname{SURE}\left(\mathrm{w}_{\mathrm{s}}, \mathrm{T}, \mathrm{k}\right)
$$

where $\mathrm{T}$ is the threshold, $\mathrm{k}$ is the window size and $\mathrm{s}$ denotes the subband. SURE is the keyword for Neigh SURE Shrink in MATLAB.

\subsubsection{Mathematical relations that are considered.}

Generally, when applying preprocessing filters, the below mathematical relations are applied to US images, it is observed that, as the noise variance increases, SNR, PSNR reduces and, mean square error and Root mean square error increases.

\section{a. Mean Square Errors (MSE)}

Mean Square Error of two images of $\mathrm{M} \times \mathrm{N}$ pixels is defined as:

$$
\operatorname{MSE}=\frac{1}{\mathrm{MN}} \sum_{\mathrm{i}=1}^{\mathrm{M}} \sum_{\mathrm{j}=1}^{\mathrm{N}}\left(\mathrm{g}_{\mathrm{i}, \mathrm{j}}-\mathrm{f}_{\mathrm{i}, \mathrm{j}}\right)
$$

Where $g(i, j)$ represents the pixel values of the original image and $f(i, j)$ represents the pixel values of the reconstructed image. For the good quality of image MSE should be low. The MSE represents the cumulative squared error between the compressed and the original image, whereas PSNR represents a measure of the peak error. To reduce the error, lower the value of RMSE is preferred. The Root mean-square error (RMSE) is square root of MSE, as defined in Equation 7. 


\section{b. Peak Signals to Noise Ratio (PSNR)}

The PSNR ratio is used as a quality measurement is computed between the two images, original and a compressed image. Generally, lower the PSNR, the quality of the compressed or reconstructed image is reduced. The PSNR is characterized by equation,

$$
\text { PSNR }=20 \log _{10} \frac{\left(2^{\mathrm{n}}-1\right)^{2}}{\mathrm{MSE}}=10 \log _{10} \frac{(255)^{2}}{\mathrm{MSE}}
$$

c. Signal to Noise Ratio (SNR)

Signal to noise ratio is used in US image to characterize the feature of image and described by the mathematical equation 9 given below.

$$
\mathrm{SNR}=10 \log _{10}\left(\frac{\sigma_{\mathrm{g}}{ }^{2}}{\sigma_{\mathrm{e}}{ }^{2}}\right)
$$

Where $\sigma_{\mathrm{g}}$ represents variance of original image and $\sigma_{\mathrm{f}}$ represents variance between original \& de-noised image. Generally, it is observed that higher the SNR, better the quality of image.

d. Mean Absolute Error (MAE)

Basically Mean absolute error (MAE) is defined as average of all absolute error

$$
\text { MAE }=\frac{\sum_{i=1}^{n} \sum_{\mathrm{j}=1}^{\mathrm{n}} \mathrm{y}(\mathrm{i}, \mathrm{j})-\mathrm{x}(\mathrm{i}, \mathrm{j})}{M N}
$$

whre $y(i, j)$ - denoised image , $x(i, j)$ input image, $M, N$ - number of rows and columns of image

\section{Results and Discussion:}

The US images were obtained from radiologist, and publically available database comprising of normal and kidney stone images in form of DICOM format, further processed using in image acquisition tool box in MATLAB $^{\circledR} 2018 \mathrm{a}$ version. During the course of processing, the associated labels with the US images were removed for better analysis and un-masked.

In next step, the resultant US image is enhanced and preprocessed to determine the statistical analysis like SNR, PSNR, RMSE, MAE for different filters discussed in earlier section. The performance analysis of US images is estimated by subjecting to different noise variance values starting from 0.01 to 0.08 , against PSNR, SNR, RMSE and MAE. It is observed that for kidney stone US images, as the noise variance increases, SNR and PSNR decreases, conversely, RMSE and MAE increases. Furthermore, any noise variance greater than 0.08 , there is indefinite decrease in SNR, and PSNR causing complexity in diagnosis of US Images.

At first the experimentation begins with loading of normal and kidney stone US Images [ Figure 3.a] for removal of background and labels associated kidney images [Figure 3.b], enhance the contrast and sharpening the quality of image [Figure 3.c] for better performance as shown.
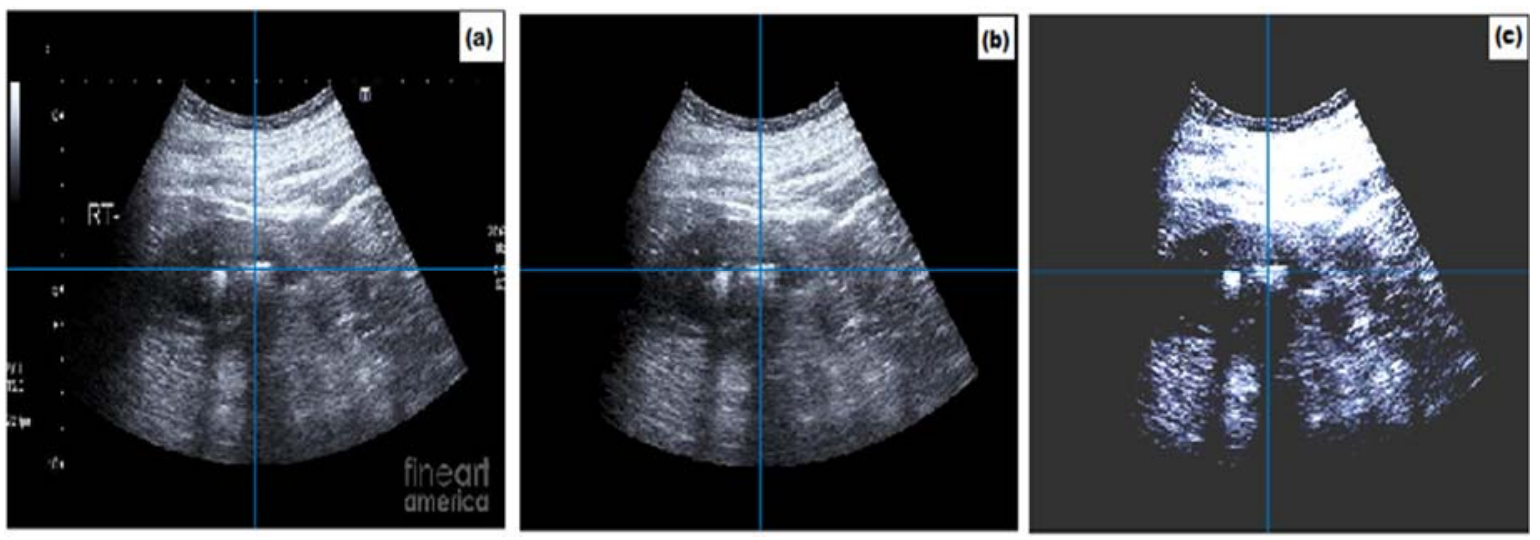

Figure 3: (a) normal kidney image. (b) Removal of background and label of kidney images. (c) Contrasted image and sharpened image

The next step is to use the discussed preprocessing filters for speckle noise reduction, allowing to applying for both normal and kidney stone US images. The three different spatial filters like, Median, Adaptive median, Weiner are used, in estimating statistical parameters. 

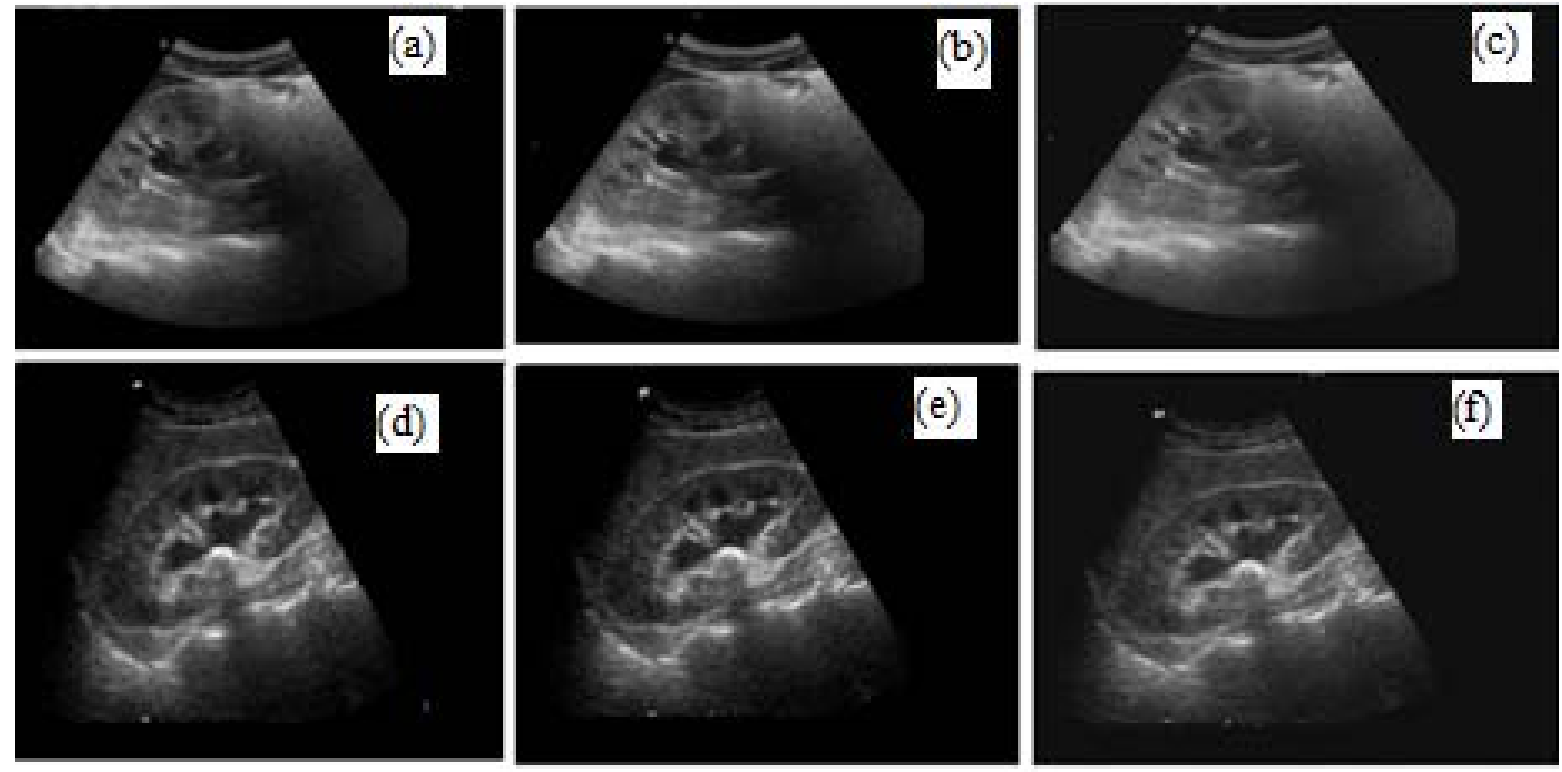

Figure 4: (a-c) results of median filter, adaptive median, Weiner filter for normal US images (d-f) results of median filter, adaptive median, Weiner filter for kidney stone US images

Figure 4 shows the preprocessed normal US images (from (a) to c)) and kidney stoneUS images (from d) to (f)). From the figure, it is evident that the speckle noise is reduced relatively well using median filter, but image is blurred and artifacts are introduced, as compared with others. In case of Adaptive filter, maximum speckle noise reduction is observed, and the edges features are well preserved. In Weiner filter, image is subjected to over enhancement, resulting in diagnosis problems and reduction in speckle noise. Likewise, for the same US images are also subjected to Neigh SURE Shrink wavelet filters with haar, db4, sym-8 and decomposition level 2 and 4 .
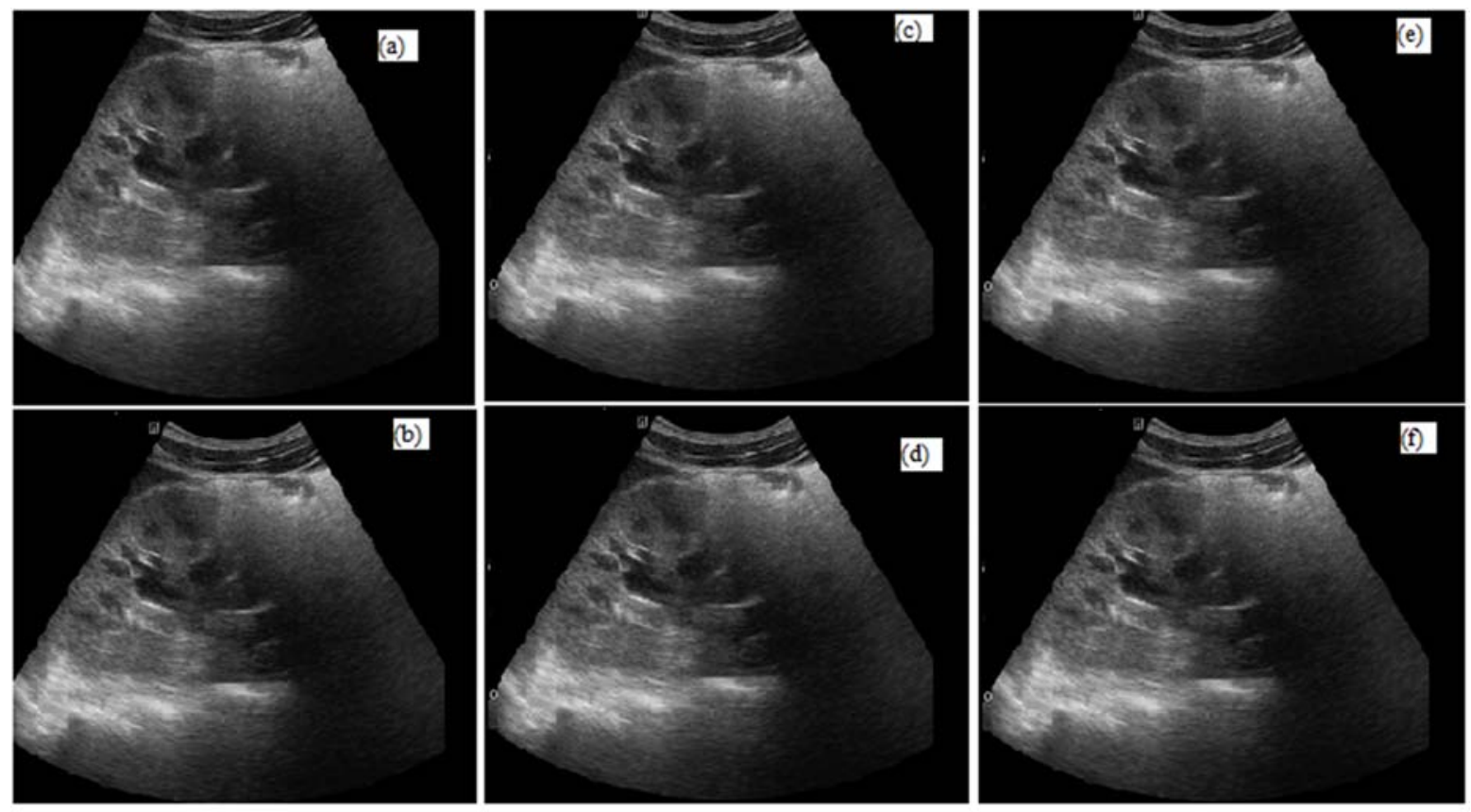

Figure 5 preprocessing filters of normal image, (a-b) Sys8 level 2 and 4 decomposition, (c-d) db4 level 2 and 4 decomposition, (e-f) haar level 2 and 4 decomposition, 

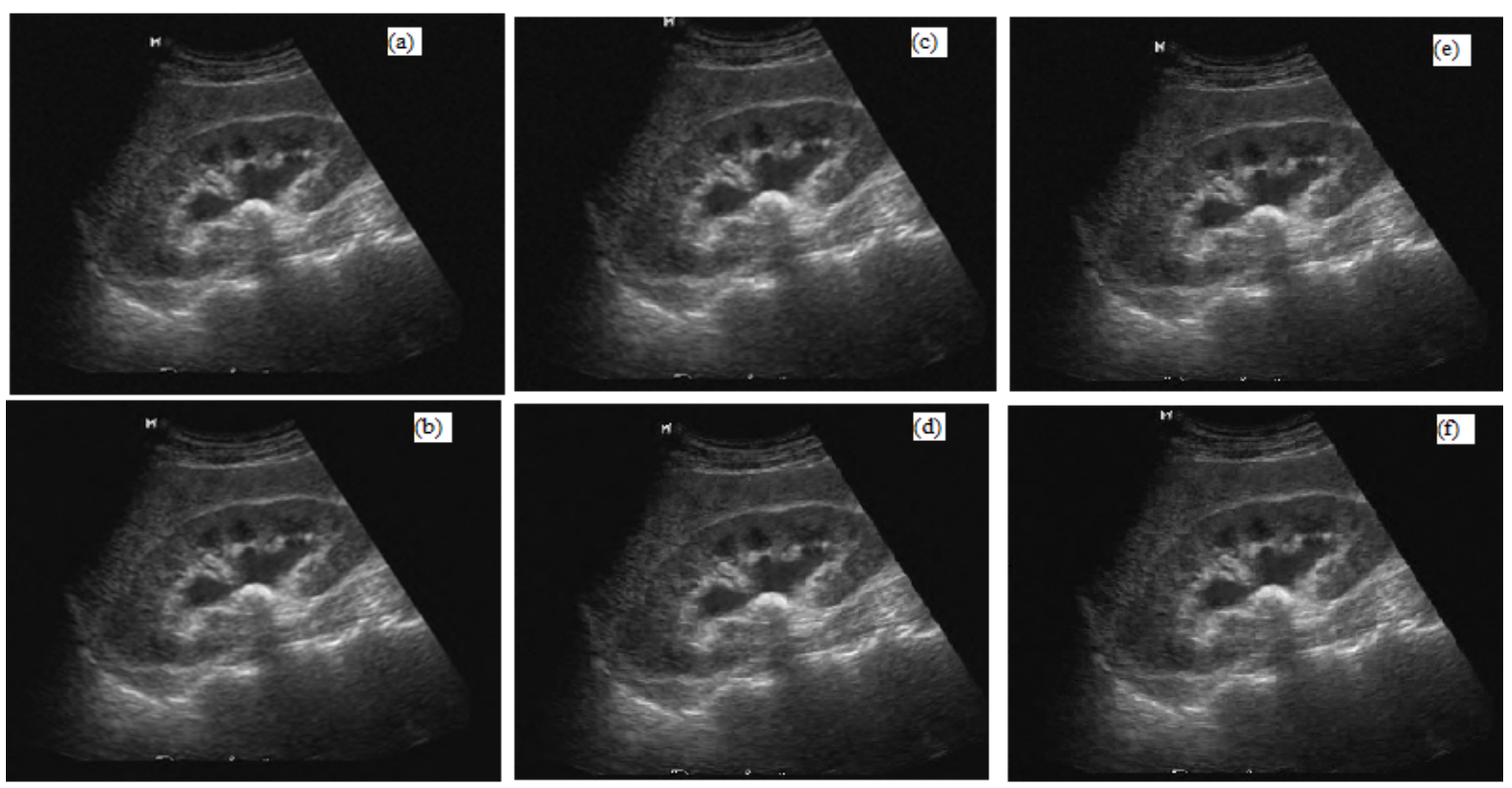

Figure 6 preprocessing filters of kidney stone US image, (a-b) Sys8 level 2 and 4 decomposition, (c-d) db4 level 2 and 4 decomposition, (ef) haar level 2 and 4 decomposition,

In the next method as shown in Figure 5 \& Figure 6 for the same normal and abnormal image, Wavelet domain filters are applied to preprocess the US image. For decomposition level-4, Wavelet Neigh sure shrink along with haar, db-4,sym- 8 , increases the contrast resolution that leads superior visual quality, in turn enhance the quality of kidney stone boundaries, reduces the speckle noise, increases the SNR, when compared to decomposition level2 , thus results in improving the image quality for further diagnosis.
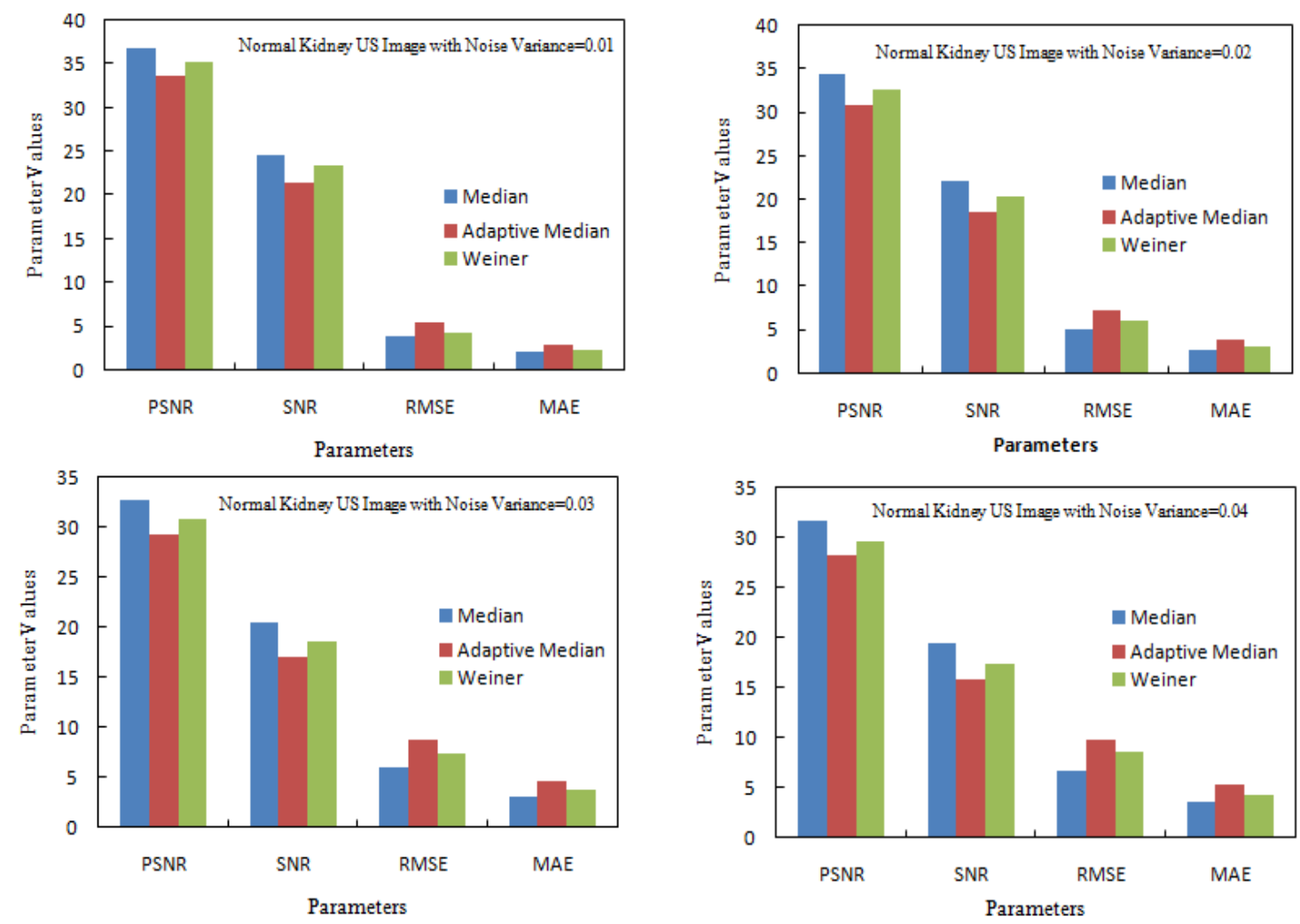

Figure 7: Statistical analysis of normal image with noise variance 0.01 to 0.04 , $\mathrm{x}$-axis in $\mathrm{dB}$ for PSNR and SNR

Upon preprocessing the US Images using different filter, the statistical parameter are estimated as shown in Figure 7, it is apparent that from Figure 8 for normal US images, for noise variance increased from 0.01 to 0.02 , PSNR is decreased by $6.66 \%$ in case of median filter, $8.25 \%$ in case of adaptive median and $7.4 \%$ in case of median filter. 
Similarly, SNR is reduced by $10 \%$ in case of median filter, $13 \%$ in case of adaptive filter and $12.7 \%$ in case of Weiner filter. Likewise, RMSE is increased by 3.25\% for median filter, $3.76 \%$ for adaptive filter, and $4 \%$ for Weiner filter. MAE is increased by $3.25 \%$ for median filter, $3.6 \%$ for adaptive filter and $3.27 \%$ for Weiner filter, leading to conclusion that, as noise variance increases PSNR, SNR decreases and RMSE and MAE increases as shown in Figure 7 \& Figure 8 for noise variance increasing from 0.01 to 0.08 .
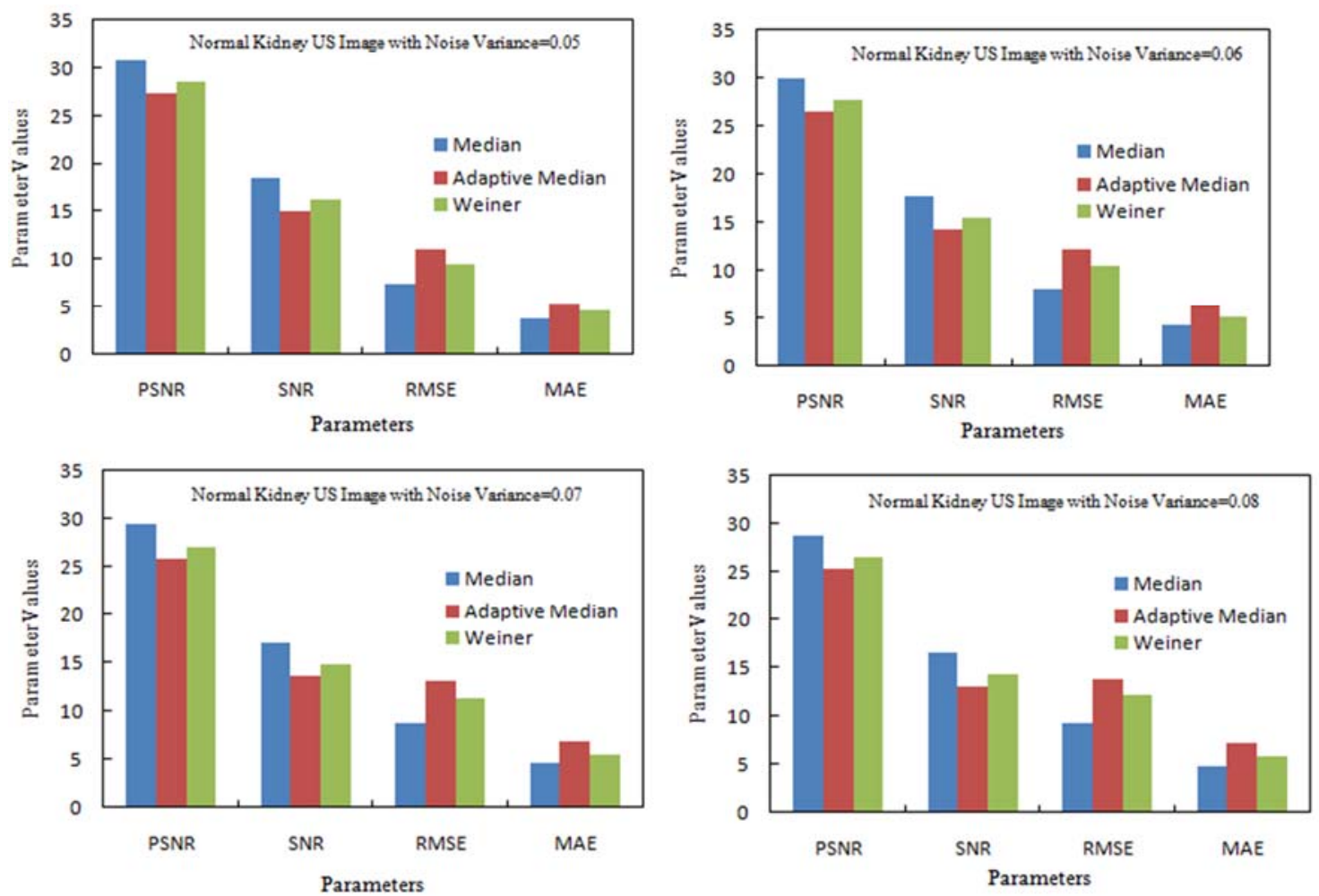

Figure 8: Statistical analysis of normal image with noise variance 0.05 to $0.08, \mathrm{x}$-axis in $\mathrm{dB}$ for PSNR and SNR
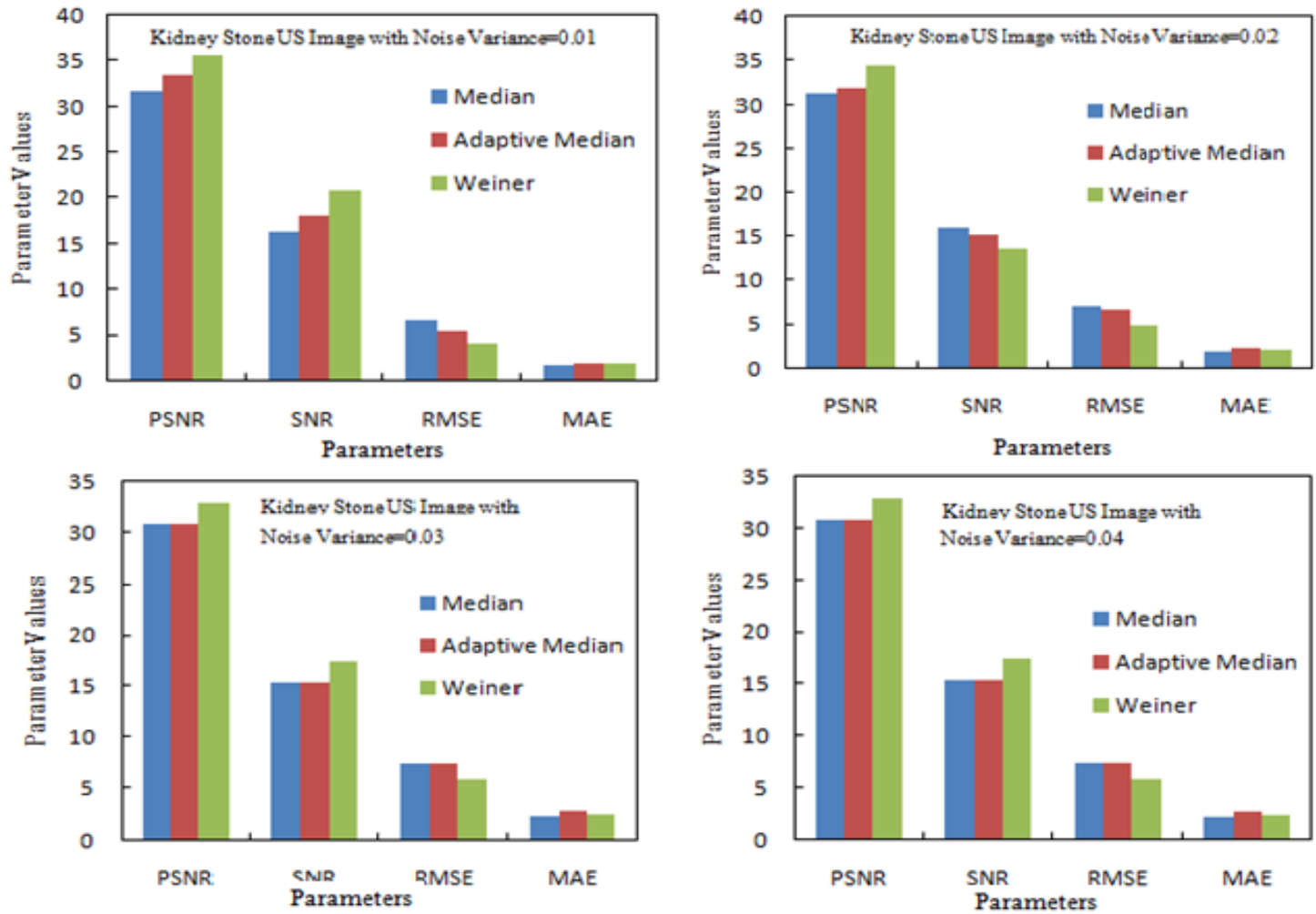

Figure 9: Statistical analysis of Kidney stone image with noise variance 0.01 to $0.04, \mathrm{x}$-axis in dB for PSNR and SNR 
Moreover, from Figure 9 for abnormal US images, for noise variance of increased from 0.01 to 0.02 , it is clear that, PSNR is decreased by $1.4 \%$ in case of median filter, $4.8 \%$ in case of adaptive median and $5.1 \%$ in case of median filter. Similarly, SNR is reduced by $2.8 \%$ in case of median filter, $1.6 \%$ in case of adaptive filter and 3.5\% in case of Weiner filter. Likewise, RMSE is increased by 5.5\% for median filter, $2.1 \%$ for adaptive filter, and $2.3 \%$ for Weiner filter. MAE is increased by $1.9 \%$ for median filter, $3.1 \%$ for adaptive filter and $1.6 \%$ for Weiner filter, thus drawing the same conclusion as that of normal images for both Figure 9 and Figure 10. Figure 11 depicts the linear variation for increase in noise value with respect to PSNR and SNR (decreasing) along with increase in RSME and MAE. Table I showcase the mean values of PSNR, SNR, RMSE and MAE for three different spatial filters of which the results the Weiner filter as preeminent. The experimental results validate to select noise variance for different quality of US images to increase SNR and PSNR. From Figure 12, it is cleared that Sym8 deco Level 4 yields better PSNR, SNR value when compare to Haar and db4 level and level 4 decomposition levels. Figure 13, shows the GUI developed using the MATLAB to detect the presence/absence the kidney stone for US images. Lastly, from the RoI and centroid, the location of kidney stone is also found to be 0.1280 centimeter.
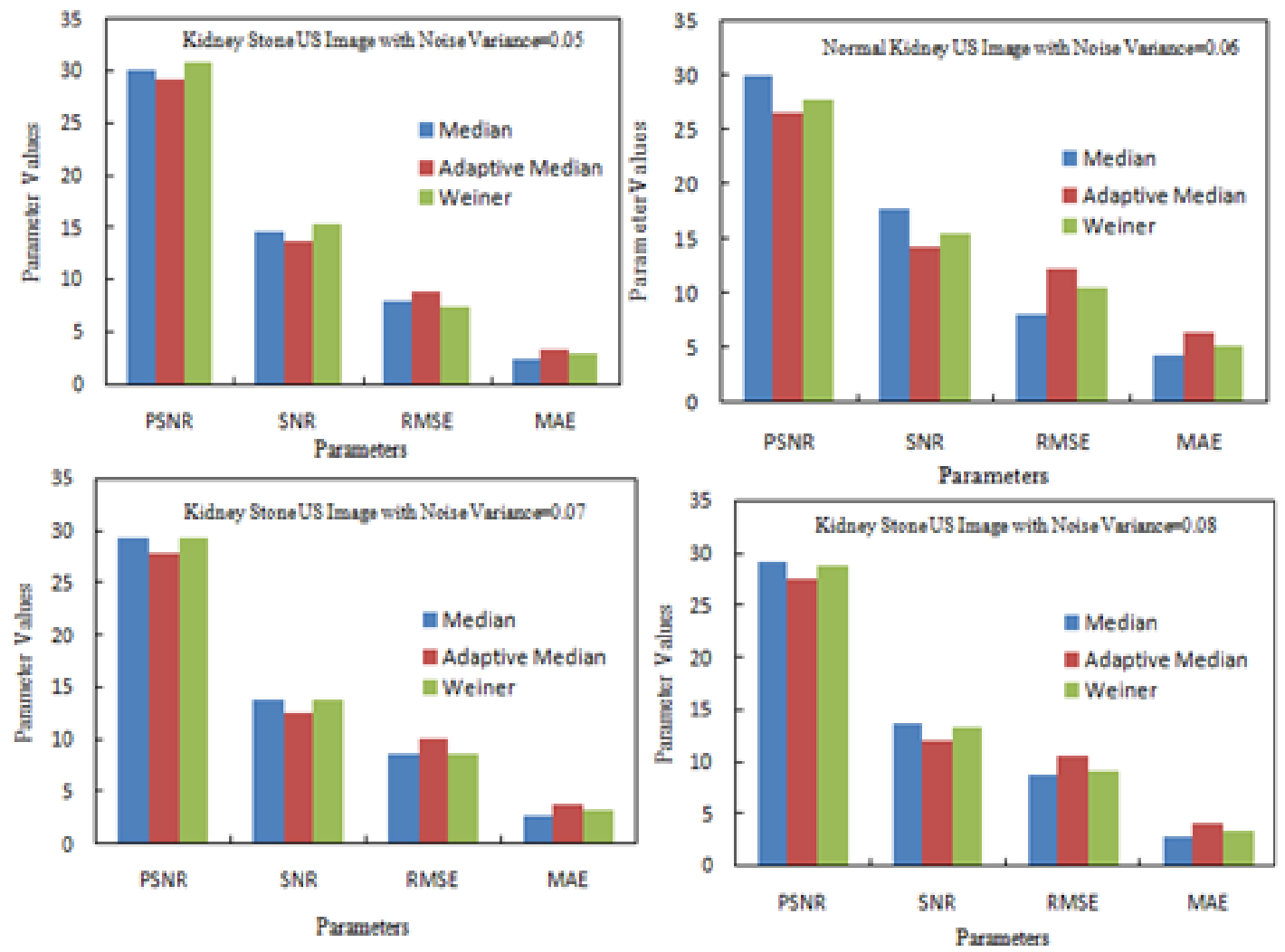

Figure 10: Statistical analysis of Kidney stone image with noise variance 0.05 to 0.08

Kidney Stone US Image with Weiner Median Filter of different Noise Variance

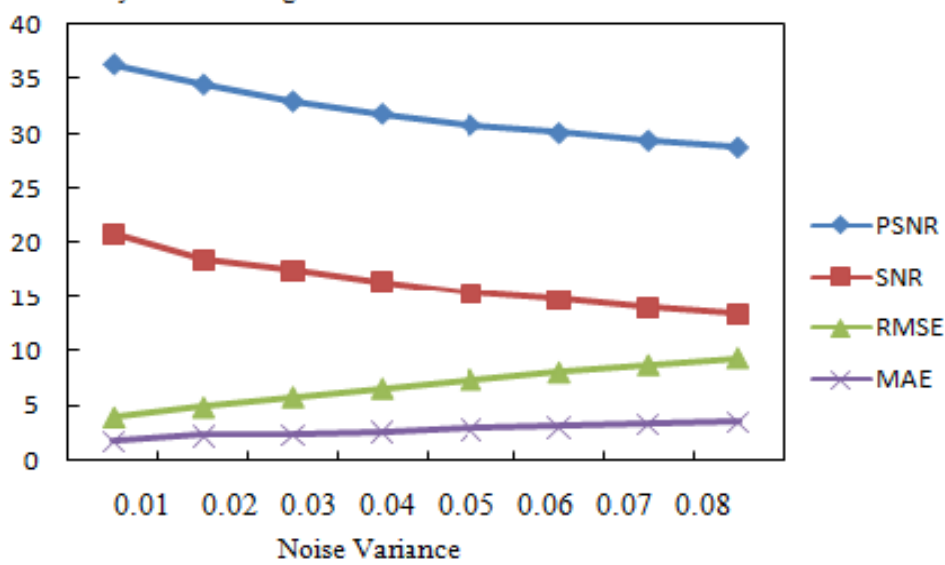

Figure 11: Statistical analysis of Normal Kidney stone image with Wavelet Haar, db4 and sym8, $\mathrm{x}$-axis in $\mathrm{dB}$ for PSNR and SNR 

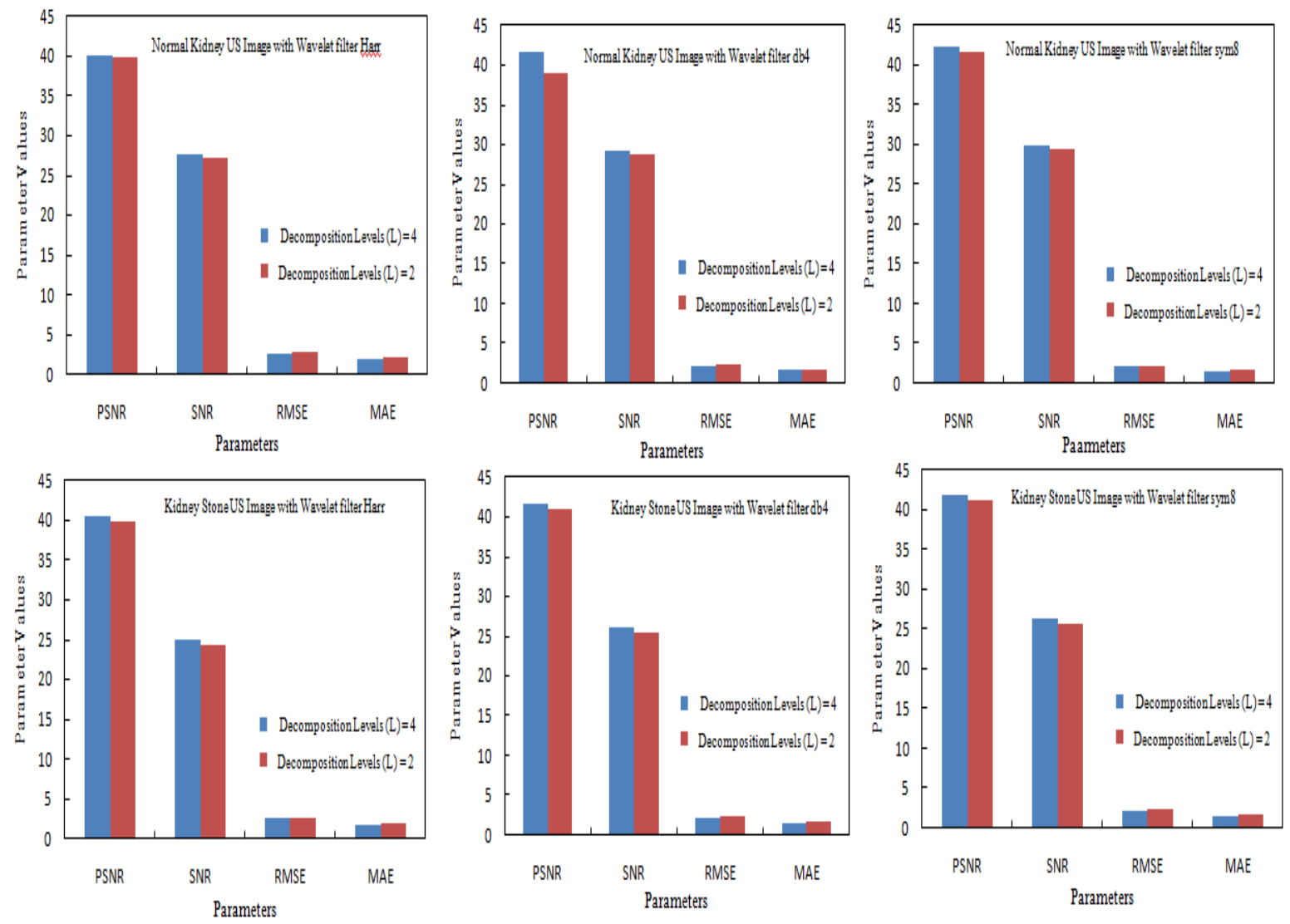

Figure 12: Statistical analysis of Normal Kidney stone image with Wavelet Haar, db4 and sym8, $\mathrm{x}$-axis in $\mathrm{dB}$ for PSNR and SNR

Table I: Mean Average of statistical parameters of kidney stone Images. All the values in $\mathrm{Db}$

\begin{tabular}{|l|l|l|l|l|}
\hline Filters & PNSR & SNR & RMSE & MAE \\
\hline Median & 30.36 & 14.86 & 7.76 & 2.23 \\
\hline Adaptive Median & 29.97 & 14.54 & 8.27 & 3.05 \\
\hline Weiner & 31.84 & 16.27 & 6.76 & 2.69 \\
\hline
\end{tabular}

\section{Conclusion}

The main focus of this work is to characterize the different value of noise parameter varying from 0.01 to 0.08 against the statistical parameters like SNR, PSNR, RMSE and MAE for different preprocessing filters like spatial and wavelet filters for US images. From preprocessing of US image with different filters, it observed with experimental results that, Sym8 decomposition level 4, provide increased SNR (26.36dB), PSNR (41.863dB), with reduction in RMSE (2.038) and MAE (1.308) as compared other filtering methods. Further, morphological operations like erosion and dilation were applied to segment the filtered US image, as obtained filtered image. By applying entropy based segmentation and morphological operations, feature extraction, RoI and exact area of kidney stone were located for in normal/ kidney stone US image. In order to assist the medical doctor for further treatment, a GUI was developed, that helps in detection of kidney stone and its area with minimum effort. 


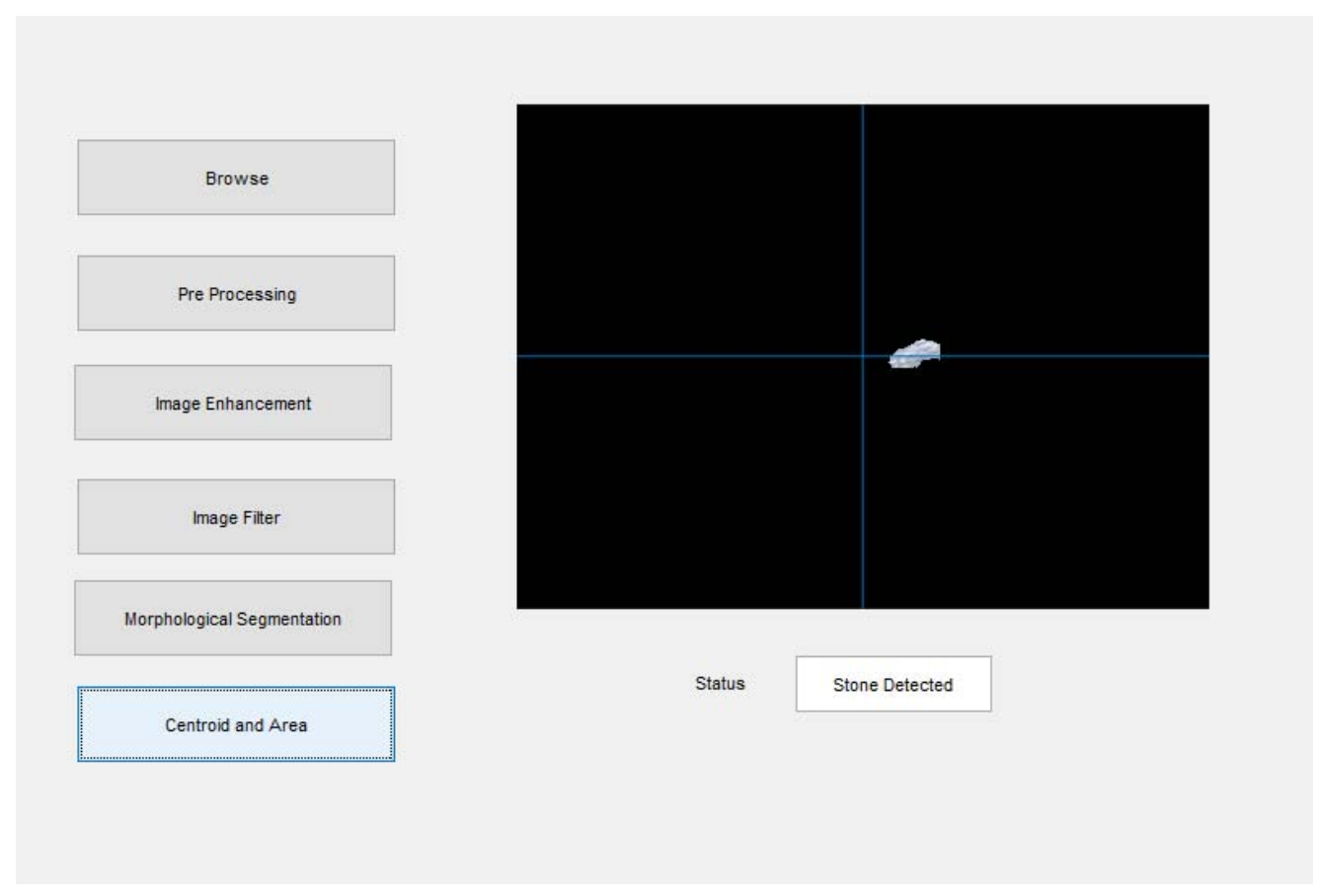

Figure 13: RoI along with Graphical User Interface (GUI) to detect the presence/absence of kidney stone

\section{References:}

[1] R.F. Wagner et al.,(1983), 'Statistics of speckle in ultrasound B-scans, IEEE Trans. Sonics Ultrasonic', vol. 30, Issue. 3, pp. 156-163.

[2] Kunio Doi. (2007), 'Computer-aided diagnosis in medical imaging: historical review, current status and future potential'. Comp Med Imaging Graphics 31(4-5), 198-211.]

[3] Summers, R. M. (2003). Road maps for advancement of radiologic computer-aided detection in the $21^{\text {st }}$ century. Radiology $229,11-13$.

[4] A.D. Hillery and R.T. Chin (1991), 'Iterative Wiener filters for image restoration', IEEE Transactions on Signal Processing Volume 39 , Issue 8, pp. $1892-1899$.

[5] Portilla et.al (2003),'Image Denoising using Scale Mixtures of Gaussians in the Wavelet Domain' IEEE Transactions on Image Processing, vol. 12, no. 11 pp. 1338-1351.

[6] Zhou qin-wu et.al,(2002)'Denoise and contrast enhancement of ultrasound speckle image based on wavelet', 6th International Conference on Signal Processing, Beijing, China, pp

[7] T. Greiner et.a 1(1991) 'Speckle reduction in ultrasonic imaging for medical applications', Volume: 1, Pages: 2993-2996.

[8] Tanzila Rahman and Mohammad Shorif Uddin,(2013), 'Speckle noise reduction and segmentation of kidney regions from ultrasound image, International Conference on Informatics, Electronics and Vision (ICIEV), DOI: 10.1109/ICIEV.2013.6572601.

[9] Kuan, D et al., (1985). Adaptive noise smoothing filters for signal dependent noise. IEEE Trans IEEE Transactions on Pattern Analysis and Machine Intelligence, Volume: PAMI-7, Issue: 2 ,pp-165-177.

[10] Lee, J. S. (1981). Refined filtering of image noise using local statistics. Elsevier, Comp Graphics Image Processing Volume 15, 380389.

[11] Frost, V et.all (1982). A model for radar image \& its application to adaptive digital filtering for multiplicative noise. IEEE Trans Pattern Analysis Machine Intelligence, Volume: PAMI-4, Issue: 2 pp-157-216.

[12] Chen, Yet al. (2003). Aggressive region growing for speckle reduction in ultrasound images. Patt Recognition Letter,Vol 24, 677-691.

[13] Huang, N.E., et.all(1998) 'The empirical mode decomposition and the Hilbert spectrum for nonlinear and non-stationary time series analysis', Proceedings of the Royal Society of London A: Mathematical, Physical and Engineering Sciences, The Royal Society, pp.903995.

[14] Gnanadurai, D. and Sadasivam, V. (2006) 'An efficient adaptive thresholding technique for wavelet based image denoising', International Journal of Signal Processing, Vol. 2, pp.114-119.

[15] Pan, Q.at.all,(1999),Two denoising methods by wavelet transform', IEEE Transactions on Signal Processing, Vol. 47, pp.3401-3406.

[16] Rahman, M.M. and Uddin, M.S. (2015) 'Optimum threshold parameter estimation of bi dimensional empirical mode decomposition using Fisher discriminant analysis for speckle noise reduction', International Arab Journal of Information Technology (IAJIT), Vol. 12, pp. $456-464$.

[17] Rafid Mostafiz, et.all,(2017) Speckle noise reduction for 3D ultrasound images by optimum threshold parameter estimation of bidimensional empirical mode decomposition using Fisher discriminant analysis, International Journal of Imaging and Robotics, Vol. 17, No. 4, pp.73-88.

[18] RamandeepKaur, et.all(2014), A New Thresholding Technique for Despeckling of Medical Ultrasound Images, IEEE Conference Publications, pp. 84-88.

[19] Tomori, Z.et.all(2003) Pyramidal seeded region growing and its use in image segmentation. Comput.Analysis of Images Pattern Lecture Notes Computer Science, Volume 1689,pp-395-402.

[20] M. B. Subramanya, et al, (2015) 'SVM-Based CAC System for B-Mode Kidney Ultrasound Images', Journal of Digital Imaging, 28(4),448-458.

[21] K.Viswanath and R. Gunasundari,(2015), Modified Distance Regularized Level Set Segmentation based analysis for Kidney stone detection Analysis, International Journal of Rough Sets and Data Analysis, 2(2), pp 22-39. 
[22] Amit Garg ; Vineet Khandelwal (2020), Segmentation-based MAP despeckling of medical ultrasound images in shearlet domain based on normal inverse Gaussian distribution, IET Image Processing, 14(4), pp 736-746.

[23] Abd-Elmoniem, et.all(2002)., Real-time speckle reduction and coherence enhancement in ultrasound imaging via nonlinear anisotropic diffusion, IEEE Transactions on Biomedical Engineering 49(9) pp-997-1014.

[24] Kang, Jinbum, et.all, (2016) ‘A New Feature-Enhanced Speckle Reduction Method Based on Multiscale Analysis for Ultrasound BMode Imaging, IEEE Transactions on Biomedical Engineering 63(6),pp- 1178-1191.

[25] Raja, K. et. all,(2008) . A hybrid fuzzy-neural system for computer-aided diagnosis of Ultrasound kidney images using prominent features," Journal of Medical Systems, vol. 32.1, pp. 65-83.

[26] Noble, J. Alison, and Djamal Boukerroui (2006). "Ultrasound image segmentation: a survey." Medical Imaging, IEEE Transactions on vol.25.8, pp.987-1010.

[27] Jun Xie; et.all (2005), "Segmentation of Kidney From Ultrasound Images based on Texture and Shape Priors", Medical Imaging, IEEE Transactions on , vol. 24.1, pp.45-57.

[28] R.M. Haralick ,and K.Shanmugam, Dinstein,(1973), Textural Features for Image Classification, Systems, Man and Cybernetics, IEEE Transactions on ,vol.6, pp.610-621.

[29] Gregorio Andria et all (2013), A Suitable Threshold for Speckle Reduction in Ultrasound Images, IEEE Transactions on Instrumentation and Measurement, Volume: 62 , Issue: 8, pp- $2270-2279$.

[30] Hyunho Choi and Jechang Jeong(2018), Despeckling Images Using a Preprocessing Filter and Discrete Wavelet Transform-Based Noise Reduction Techniques, IEEE Sensors Journal Vol18, Issue: 8 , pp- $3131-3139$. 